SINP-2001-37/677

\title{
String-Loop Corrected Magnetic Black Holes
}

\author{
Mikhail Z. Iofa ${ }^{1}$ \\ Skobeltsyn Institute of Nuclear Physics \\ Moscow State University \\ Moscow 119899, Russia
}

December 24, 2018

\begin{abstract}
We discuss the form of the string-loop-corrected effective action obtained by compactification of the heterotic string theory on the manifold $K 3 \times T^{2}$ or on its orbifold limit and the loop-corrected magnetic black hole solutions of the equations of motion. Effective $4 \mathrm{D}$ theory has $\mathrm{N}=2$ local supersymmetry. Using the string-loop-corrected prepotential of the $\mathrm{N}=2$ supersymmetric theory, which receives corrections only from the string world sheets of torus topology, we calculate the loop corrections to the tree-level gauge couplings and solve the loop-corrected equations of motion. At the string-tree level, the effective gauge couplings decrease at small distances from the origin, and in this region string-loop corrections to the gauge couplings become important. A possibility of smearing the singularity of the tree-level supersymmetric solution with partially broken supersymmetry by quantum corrections is discussed.
\end{abstract}

\footnotetext{
1iofa@theory.sinp.msu.ru
} 


\section{Introduction}

At present, string theory is considered the best candidate for a fundamental theory that would provide a consistent quantum theory of gravity unified with the other interactions [1]. In particular, string theory provides a powerful approach to the physics of black holes (review and further refs. in $[2,3])$. In this setting, we meet a fundamental problem of understanding how the intrinsically stringy effects modify the Einstein gravity.

In this paper we discuss two of these effects: presence of scalar fields such as the dilaton and moduli, and higher-genus contributions modifying the tree-level effective action. We focus on higher-genus corrections, because string theory, being a theory formulated on a world sheet, always contains string-loop corrections from higher topologies of the world sheet (for the twoderivative terms these vanish for higher supersymmetries $N \geq 4$ ), while $\alpha^{\prime}$ corrections can vanish in certain constructions based on conformal field theories and for a large class of backgrounds $[4,5]$.

We consider the perturbative one-string-loop (torus topology) corrections for a special class of backgrounds: $4 D$ black holes provided by the "chiral null models" [4,5] embedded in heterotic string theory compactified on the manifold $K 3 \times T^{2}$. For this compactification pattern, the effective $4 D$ field theory is described by $N=2$ supergravity interacting with matter.

The universal sector contains supergravity and vector multiplets, the vector components resulting from dimensional reduction of $6 D$ metric and the antisymmetric tensor on the two-torus $T^{2}$. By using the (perturbative) one-loop-corrected prepotential in the $N=2$ supergravity, we calculate the effective gauge couplings in the universal sector of the theory. Due to the $N=2$ supersymmetry, there are no loop corrections to the prepotential beyond the one loop [6, 7, 8].

In string perturbation theory, higher order contributions enter with the factor $e^{-\frac{1}{2} \chi \phi^{\prime}}$, where $\chi$ is the Euler characteristic of the string world sheet. The exponent $e^{\phi_{\infty}} \equiv \epsilon$, where $\phi_{\infty} \equiv$ $\left.\phi\right|_{|x| \rightarrow \infty}$ can be considered as a string-loop expansion parameter ${ }^{2}$. The dilaton $\phi^{\prime}$ is split as $\phi+\phi_{\infty}$.

In the case of magnetic black hole with the charges $P_{1}$ and $P_{2}$ the string-tree-level dilaton $\phi=\frac{1}{2} \ln \frac{\left(r+P_{1}\right)\left(r+P_{2}\right)}{r^{2}}$ increases, and the tree-level gauge couplings of the vector fields in the universal sector which are proportional to $e^{-\phi}$ decrease at small distances, so that the effective gauge couplings are sensitive to string-loop corrections.

The loop-corrected configuration of magnetic black hole can be obtained by solving either the equations of motion derived from the loop-corrected effective action, or spinor Killing equations (conditions for supersymmetry variations of spinors to vanish) ( review and refs. in [9]).

In this paper we follow the first way, i.e. solve the loop-corrected equations of motion. Solution of spinor Killing equations is presented elsewhere [10]. Equations of motion are solved analytically in the first order in the loop-counting parameter $\epsilon$. Considerable technical simplification is achieved for a special choice of magnetic charges, in which case the the tree-level metric of the two-torus is independent of coordinates. However, qualitatively, the results remain unchanged for unequal charges.

Solving the system of the equations of motion for the moduli and the Einstein-Maxwell equations in the first order in $\epsilon$, we obtain the loop-corrected metric and dilaton. A family of

\footnotetext{
${ }^{2}$ For closed surfaces without boundaries, $\chi=2-2 g$ is the number of handles. The factor $e^{\phi_{\infty}}$ multiplying the tree-level effective action is absorbed in the Newton coupling constant.
} 
solutions for the metric of the loop-corrected magnetic black hole is

$$
g_{i i}=-g^{00}=\left(1+\frac{P}{r}\right)+\epsilon\left(A_{1} \frac{P}{r}-A_{2} \frac{P}{r+P}\right) \text {, }
$$

where $A_{i}$ are arbitrary constants, $P=8 \sqrt{P^{1} P^{2}}$. Supersymmetry fixes the value of $A_{i}$ : $A_{1}=-\frac{P h}{2 T U}, A_{2}=0$, where $h(T, U)$ is the real (unambiguous) part of the one-string-loop correction to the prepotential, $T, U$ are the standard string tree-level moduli. For a particular compactification of the heterotic string for which the loop correction to the prepotential was calculated it appears to be strictly negative [19]. The above metric can be considered as the first term in the expansion in the loop-counting parameter of the metric

$$
g_{i i}=-g^{00}=1+\frac{P}{r+\epsilon \frac{r+|\epsilon P H|}{r+P}} .
$$

When extrapolated to the region of small $r$, this metric has no singularity, which is smeared by quantum corrections.

In Sect. 2 we review the structure of the $4 \mathrm{D}$ magnetic black hole in heterotic string theory provided by chiral null model.

In Sect. 3 we discuss the $N=2$ locally supersymmetric effective theory.

Using the symplectic structure of the theory, we calculate the gauge couplings in a basis admitting the prepotential and by symplectic transformation obtain them in the basis associated with the heterotic string compactification (in which case the prepotential does not exist) and construct the loop-corrected action.

In Sect.4 we solve the loop-corrected equations of motion. Starting from the tree-level extremal magnetic black-hole solution, in the first order in string coupling constant, we obtain an analytic solution of the loop-corrected equations of motion.

Solution of the loop-corrected equations of motion and calculation of the loop-corrected gauge couplings is presented in the Appendixes.

\section{Charged 4D black hole solutions in 4D effective field theory.}

The effective field theory which describes dynamics of the light fields in four dimensions depends on the pattern of compactification of the initial superstring theory. Heterotic string theory, when compactified on the manifold $K 3$ or on a suitable orbifold yields locally supersymmetric $6 D$ theory with $N=1$ supersymmetry, while compactification of the heterotic string on the 4-torus $T^{4}$ results in $6 D, N=2$ locally supersymmetric theory.

The bosonic part of the universal sector for both compactifications has the same form

$$
I_{6}=\frac{1}{2 \kappa_{6}{ }^{2}} \int d^{6} x \sqrt{-G} e^{-\Phi^{\prime}}\left[R+\left(\partial \Phi^{\prime}\right)^{2}-\frac{H^{2}}{12}\right]+\ldots
$$

A large class of solutions to the equations of motion following from the action (1) is provided by the chiral null models $[4,5,12]$. From the string-theoretical point of view, a chiral null 
model is a conformal 2D theory interpreted as string world-sheet Lagrangian with nontrivial backgrounds:

$$
L=F(x) \partial u\left[\bar{\partial} v+K(u, x) \bar{\partial} u+2 \mathcal{A}_{i}(u, x) \bar{\partial} x^{i}\right]+\left(G_{i j}+B_{i j}\right)(x) \partial x^{i} \bar{\partial} x^{j}+\mathcal{R} \Phi(x) .
$$

As solutions of the equations of motion of the full effective theory, the backgrounds ensure vanishing of the $\beta$-functions and conformal invariance of the corresponding $2 \mathrm{D}$ theory.

An important special case of chiral null models (2) is provided by the chiral null models with curved transverse part of the form $[4,5,12]$

$$
\begin{gathered}
L=F(x) \partial u(\bar{\partial} v+K(x) \bar{\partial} u)+f(x) k(x)\left[\partial x^{4}+a_{s}(x) \partial x^{s}\right]\left[\bar{\partial} x^{4}+a_{s}(x) \bar{\partial} x^{s}\right] \\
+f(x) k^{-1}(x) \partial x^{s} \bar{\partial} x^{s}+b_{s}(x)\left(\partial x^{4} \bar{\partial} x^{s}-\bar{\partial} x^{4} \partial x^{s}\right)+\frac{1}{8} \alpha^{\prime} \sqrt{g^{(2)}} R^{(2)} \ln F(x) f(x),
\end{gathered}
$$

Here $x^{s}=\left(x^{1}, x^{2}, x^{3}\right)$ and $v=2 t$ are non-compact space-time coordinates, $u=y^{2}$ and $x^{4}=y^{1}$ are compact toroidal coordinates. Written in $4 D$ form, the Lagrangian of the chiral null model is

$$
\begin{gathered}
L=\left(G_{\mu \nu}^{\prime}+B_{\mu \nu}^{\prime}\right)(x) \partial x^{\mu} \bar{\partial} x^{\nu}+G_{m n}(x)\left[\partial y^{m}+A_{\mu}^{(1) m}(x) \partial x^{\mu}\right]\left[\bar{\partial} y^{n}+A_{\nu}^{(1) n}(x) \bar{\partial} x^{\nu}\right] \\
+A_{n \mu}^{(2)}(x)\left(\partial y^{n} \bar{\partial} x^{\mu}-\bar{\partial} y^{n} \partial x^{\mu}\right)+\frac{1}{8} \alpha^{\prime} \sqrt{g^{(2)}} R^{(2)} \Phi^{\prime}(x) .
\end{gathered}
$$

The background fields are independent of compact coordinates and can be interpreted as solutions of the equations of motion following from the action of the $4 \mathrm{D}$ effective field theory obtained by dimensional reduction from the $6 \mathrm{D}$ action (1)[13]

$$
I_{4}=\frac{1}{2 \kappa_{4}^{2}} \int d^{4} x \sqrt{-G^{\prime}} e^{-\phi^{\prime}}\left[R+\left(\partial \phi^{\prime}\right)^{2}-\frac{\left(H^{\prime}\right)^{2}}{12}-\frac{1}{4} F(L M L) F+\frac{1}{8} \operatorname{Tr}(\partial M L \partial M L)+\ldots\right] .
$$

Here $G=\left(G_{m n}\right), B=\left(B_{m n}\right), \quad m, n=1,2$ and the space-time tensor indices in (5) are raised with respect to the metric $G_{\mu \nu}^{\prime}$.

$$
M=\left(\begin{array}{cc}
G^{-1} & G^{-1} B \\
-B G^{-1} & G
\end{array}\right), \quad L=\left(\begin{array}{cc}
0 & I_{2} \\
I_{2} & 0
\end{array}\right) .
$$

The $4 D$ string-frame backgrounds are related to the fields in the Lagrangian (3) as [13]

$$
\begin{aligned}
& G_{\mu \nu}^{\prime}=G_{\mu \nu}-G_{m n} A_{\mu}^{(1) m} A_{\nu}^{(1) n}, \quad B_{\mu \nu}^{\prime}=B_{\mu \nu}-B_{m n} A_{\mu}^{(1) m} A_{\nu}^{(1) n}, \\
& H_{\mu \nu \lambda}^{\prime}=H_{\mu \nu \lambda}-\left(A^{(1) n} H_{n \nu \lambda}-A^{(1) m} A^{(1) n} H_{m n \lambda}+\text { cycl.perms. }\right) .
\end{aligned}
$$

The gauge fields and dilaton are

$$
A_{\mu}^{(1) n}=G^{n m} G_{m \mu}, \quad A_{n \mu}^{(2)}=B_{n \mu}, \quad \phi^{\prime}=\Phi^{\prime}-\frac{1}{2} \ln G, \quad G \equiv \operatorname{det} G_{m n},
$$

and their explicit form can be read off from (3):

$$
A_{\mu}^{(1) 1}=\left(0, a_{s}\right), \quad A_{\mu}^{(1) 2}=\left(K^{-1}, 0\right), \quad A_{1 \mu}^{(2)}=\left(0, b_{s}\right), \quad A_{2 \mu}^{(2)}=(F, 0), \quad B_{m n}=B_{\mu \nu}=0 .
$$


The vectors $a_{s}$ and $b_{s}$ are expressed via the functions $f$ and $k^{-1}$ which are solutions of the harmonic equation.

The dilaton $\Phi^{\prime}$ in (1) can be split in the sum $\Phi+\Phi_{\infty}$, where at spatial infinity $\Phi=0$, and $\Phi_{\infty}$ is a free parameter, and also $\phi^{\prime}=\phi+\phi_{\infty}$.

Written in the Einstein frame, where $g_{\mu \nu}=e^{-\phi} G_{\mu \nu}^{\prime}$, the action (5) is [13]

$$
I_{4}=\int d^{4} x \sqrt{-g}\left[R-\frac{1}{2}(\partial \phi)^{2}-\frac{e^{-\phi}}{4} \mathcal{F}(L M L) \mathcal{F}+\frac{a_{1}}{4 \sqrt{-g}} \mathcal{F} L^{*} \mathcal{F}+\frac{1}{8} \operatorname{Tr}(\partial M L \partial M L)+\ldots\right] .
$$

Here we kept only the non-constant part of the dilaton $\phi$ and introduced the axion

$$
\partial_{\rho} a_{1}=-H^{\prime \mu \nu \lambda} e^{-2 \phi} \sqrt{-g} e_{\mu \nu \lambda \rho}
$$

A class of solutions of the equations of motion derived from the action (8) is [5, 14] (only non-vanishing backgrounds are presented)

$$
\begin{aligned}
& d s^{2}=-\Lambda(r) d t^{2}+\Lambda^{-1}\left(d r^{2}+r^{2} d \Omega_{2}^{2}\right), \\
& \Lambda^{2}(r)=F K^{-1} k f^{-1}, \\
& \phi=\frac{1}{2} \ln F K^{-1} f k^{-1}, \\
& G_{11}=F K, \\
& G_{22}=f k,
\end{aligned}
$$

where

$$
K=A\left(+\frac{Q_{1}}{r}\right), \quad F^{-1}=B\left(1+\frac{Q_{2}}{r}\right), \quad k^{-1}=a\left(1+\frac{P_{1}}{r}\right), \quad f=b\left(1+\frac{P_{2}}{r}\right)
$$

are harmonic functions. Demanding that at spatial infinity the metric and dilaton are asymptotic to the Lorentzian metric and unity, we have $A B a b=1$ and $\frac{A B}{a b}=1$, which is solved by $A B=1$ and $a b=1 . F^{(1) 1}$ and $F_{1}^{(2)}$ are magnetic and $F^{(1) 2}$ and $F_{2}^{(2)}$ are electric field strengths. $G_{11}$ and $G_{22}$ are the nonzero components of the metric of the torus $T^{2}$ on which are compactified two dimensions of the six-dimensional theory. From the $4 D$ point of view, the backgrounds are interpreted as charged black holes.

\section{Loop-corrected 4D effective field theory.}

Compactification of heterotic string theory on the manifold $K 3$ or on a suitable orbifold yields $6 D$ theory with $N=1$ supersymmetry. Further compactification on a two-torus yields a $N=2$, $4 D$ locally supersymmetric theory.

To investigate solutions of the loop-corrected equations of motion in this theory, we make use of the well-developed technique of the $N=2$ supersymmetric supergravity $[7,15,16,17,18]$. The form of $N=2$ supergravity depends on the choice of a holomorphic vector bundle $\left(X^{A}, F_{A}\right)$, where $X^{A}$ denote both the vector superfields and the complex scalar components of these superfields. The moduli and the vector fields are combined in $N=2$ vector multiplets. If 
a holomorphic vector bundle admits a holomorphic prepotential $F(X)$, the latter completely defines dynamics of $N=2$ supersymmetric theory. In particular, in this case $F_{A}=\partial F / \partial X^{A}$.

In the heterotic string compactification the prepotential has no perturbative loop corrections beyond one loop and is of the form $[6,7,8,11,19]$

$$
F=-\frac{X^{1} X^{2} X^{3}}{X^{0}}-i X^{0^{2}} \epsilon h^{(1)}\left(-i \frac{X^{2}}{X^{0}},-i \frac{X^{3}}{X^{0}}\right)+\ldots,
$$

where dots stand for contribution of other moduli.

The moduli space is parametrized by complex coordinates $z_{i}=i y_{i}$. Special coordinates are introduced as

$$
\frac{X^{i}}{X^{0}}=i y_{i}=\left(\operatorname{Re} y_{i}+i a_{i}\right), \quad X^{0}=1
$$

We use the standard identification of the tree-level special coordinates

$$
\begin{aligned}
& y_{1}=S=i\left(e^{-\phi}+i a_{1}\right), \\
& y_{2}=T=i\left(\sqrt{G}+i B_{12}\right), \\
& y_{3}=U=i\left(\frac{\sqrt{G}+i G_{12}}{G_{22}}\right) .
\end{aligned}
$$

In the case of solution (9), $B_{12}=G_{12}=a_{1}=0$.

In the perturbative approach, we neglect non-perturbative corrections to the prepotential of the form $f\left(e^{-2 \pi S}, T, U\right)$. The loop corrections are accompanied by a factor $\epsilon$ and are treated perturbatively.

The bosonic part of the universal sector of the $N=2$ supersymmetric theory written in the standard form of $N=2$ special geometry [15, 16, 17, 18, 20]. in the holomorphic section with the prepotential is

$$
I_{4}=\int d^{4} x \sqrt{-g}\left[-\frac{1}{2} R+i\left(\bar{N}_{I J} \mathcal{F}^{-I} \mathcal{F}^{-J}-N_{I J} \mathcal{F}^{+I} \mathcal{F}^{+J}\right)+k_{i \bar{j}} \partial_{\mu} y_{i} \partial^{\mu} \bar{y}_{\bar{j}}+\ldots\right]
$$

Here $N_{I J}$ are the gauge couplings

$$
N_{I J}=\bar{F}_{I J}+2 i \frac{\left(I m F_{I K} X^{K}\right)\left(I m F_{J L} X^{L}\right)}{\left(X^{I} I m F_{I J} X^{J}\right)},
$$

where $F_{I J}=\partial^{2} F / \partial X^{I} \partial X^{J}$ and $F$ is the total loop-corrected prepotential, and (anti)self-dual field strengths are

$$
\mathcal{F}_{\mu \nu}^{ \pm}=\frac{1}{2}\left(\mathcal{F}_{\mu \nu} \pm \sqrt{-g} \mathcal{F}_{\mu \nu}^{*}\right)
$$

where $\mathcal{F}_{\mu \nu}^{*}=\frac{1}{2} e_{\mu \nu \rho \lambda} \mathcal{F}^{\rho \lambda}$, and $e_{\mu \nu \rho \lambda}$ is the flat antisymmetric tensor.

The vector fields are re-labeled in correspondence with the moduli with which they form the supermultiplets

$$
A_{\mu}^{1}=\mathcal{A}_{\mu}^{0}, \quad B_{1 \mu}=\mathcal{A}_{\mu}^{1}, \quad A_{\mu}^{2}=\mathcal{A}_{\mu}^{2}, \quad B_{2 \mu}=\mathcal{A}_{\mu}^{3} .
$$

The Kaehler metric $k_{i \bar{j}}$ is expressed through the Kaehler potential

$$
K=-\log i\left(\bar{X}^{A} F_{A}-X^{A} \bar{F}_{A}\right) .
$$


The dilaton kinetic term and last term in the tree-level action (8) $\frac{1}{8} \operatorname{Tr}(\partial M L \partial M L)$ can be written in terms of the tree-level Kahler potential

$$
K^{(0)}=-\log [(T+\bar{T})(U+\bar{U})(S+\bar{S})
$$

as

$$
k_{i \bar{j}}^{(0)} \partial_{\mu} y_{i} \partial^{\mu} \bar{y}_{\bar{j}}=K_{S \bar{S}}^{(0)} \partial S \partial \bar{S}+K_{T \bar{T}}^{(0)} \partial T \partial \bar{T}+K_{U \bar{U}}^{(0)} \partial U \partial \bar{U} .
$$

The loop-corrected Kaehler potential calculated by using the loop-corrected prepotential (10) is of the form $[6,7,11]$

$$
K=-\log [(T+\bar{T})(U+\bar{U})(S+\bar{S}+\epsilon V(T, \bar{T}, U, \bar{U})],
$$

where the Green-Schwarz function $V$ is

$$
V=\frac{\operatorname{Re} h^{(1)}-\operatorname{Re} T \operatorname{Re} \partial_{T} h^{(1)}-\operatorname{Re} U \operatorname{Re} \partial_{U} h^{(1)}}{\operatorname{Re} T \operatorname{Re} U} .
$$

Beyond the tree level the dilaton mixes with other moduli. At the one-loop level

$$
S=e^{-\phi}-\frac{V}{2}+i a_{1}
$$

The kinetic terms for the moduli calculated with the prepotential (10) are

$$
4 \frac{\partial^{2} K}{\partial y_{i} \partial \bar{y}_{\bar{j}}} \partial_{\mu} z^{i} \partial^{\mu} \bar{z}^{\bar{j}}=(\partial \phi)^{2}+\frac{\left|\partial y_{a}\right|^{2}}{\left(R e y_{a}\right)^{2}}+e^{2 \phi}\left(\partial_{\mu} a_{1}+X_{\mu}\right)^{2}+\frac{e^{\phi}}{2} V_{y_{a} \bar{y}_{b}} \partial y_{a} \partial \bar{y}_{b} .
$$

Here

$$
i X_{\mu}=\frac{1}{2}\left(V_{\bar{y}_{a}} \partial_{\mu} \bar{y}_{a}-V_{y_{a}} \partial_{m} y_{a}\right) .
$$

The loop-corrected universal part of the heterotic string effective action can be written as

$$
\begin{aligned}
I_{4}= & \int d^{4} x \sqrt{-g}\left[-\frac{1}{2} R+i\left(\bar{N}_{I J} \mathcal{F}^{-I} \mathcal{F}^{-J}-N_{I J} \mathcal{F}^{+I} \mathcal{F}^{+J}\right)+\frac{1}{4}\left((\partial \phi)^{2}+e^{2 \phi}\left(\partial a_{1}+X\right)^{2}\right)\right. \\
& \left.+\frac{|\partial T|^{2}}{(R e T)^{2}}+\frac{|\partial U|^{2}}{(R e U)^{2}}+\frac{e^{\phi}}{2}\left(V_{T \bar{T}}|\partial T|^{2}+V_{U \bar{U}}|\partial U|^{2}+V_{U \bar{T}} \partial U \partial \bar{U}+V_{T \bar{U}} \partial T \partial \bar{U}\right)+\ldots\right]
\end{aligned}
$$

Starting with the compactified heterotic string theory, one naturally arrives at the effective action written in terms of the "heterotic" vector bundle. In this holomorphic section the moduli are treated non-symmetrically, the modulus $S$ playing the role of the coupling constant $[7,21,22]$, and the vector couplings become weak in the large-dilaton limit. It is known that in terms of the heterotic vector bundle it is impossible to introduce a prepotential, however, one can calculate the gauge couplings in this holomorphic section by performing a symplectic transformation from the section with the prepotential [7, 16]. Following [7], the heterotic holomorphic section $(\hat{X}, \hat{F})$ is obtained from that admitting the prepotential by performing symplectic transformation

$$
\left(\begin{array}{c}
\hat{X} \\
\hat{F}
\end{array}\right)=O\left(\begin{array}{c}
X \\
F
\end{array}\right) \quad O=\left(\begin{array}{cc}
U & Z \\
W & V
\end{array}\right),
$$

where the nonzero elements of the matrix $O$ are $U_{J}^{I}=V_{J}^{I}=\delta_{J}^{I}$ for $I, J \neq 1, W_{11}=-Z^{11}= \pm 1$. The gauge coupling constants transform as

$$
\hat{N}=(V N+W)(U+Z N)^{-1} .
$$




\section{Equations of motion}

We look for a static spherically-symmetric solution to the equations of motion derived from the loop-corrected effective action with the final goal to find the loop-corrected dilaton and metric. In magnetic case, the metric and dilaton have singular behavior at the origin, while other background fields stay constant, and only for the former we can expect qualitative effects of loop corrections.

We shall concentrate on the case of tree-level solutions which are purely magnetic $\left(Q_{1}=\right.$ $Q_{2}=0$ ) extremal black holes with equal magnetic charges $P_{1}=P_{2}=P$. These solutions can be embedded in $4 D N=2$ dilatonic supergravity and leave $1 / 2$ of the supersymmetry unbroken. In this case, the non-vanishing backgrounds of the chiral null model are expressed via a single function (we consider one-center solution) $f_{0}$ :

$$
\begin{array}{r}
d s^{2}=-f_{0}^{-1} d t^{2}+f_{0} d x^{i^{2}}, \quad f_{0}(r)=1+\frac{P}{r}, \\
\phi=\ln f_{0}, \quad a_{\varphi}=a^{-1} P(1-\cos \vartheta), \quad b_{\varphi}=a P(1-\cos \vartheta),
\end{array}
$$

where $a_{\varphi}$ and $b_{\varphi}$ are nonzero components of potentials in spherical coordinates. The tree-level components of the internal metric $G_{m n}$ are

$$
G_{11}=a^{2}, \quad G_{22}=A^{2} .
$$

The magnetic field strengths are

$$
F_{i j}^{(1) 1}=a^{-1} F_{i j}, \quad F_{1 i j}^{(2)}=a F_{i j}, \quad F_{i j}=-\varepsilon_{i j k} \partial^{k} f_{0},
$$

and in spherical coordinates have a single nonzero component $F_{\vartheta \varphi}=P \sin \vartheta$.

At the tree level, in the case of magnetic black hole solution, the imaginary parts of the moduli, $a_{i}$, vanish, and, if appear at the one-loop level, are of order $O(\epsilon)$. With the accuracy of the terms of higher order in $O(\epsilon)$, the imaginary parts of the moduli $S, T$ and $U$ do not enter the equations for the real parts.

It is convenient to introduce the functions $\gamma$ and $\sigma$ as

$$
\operatorname{ReT}=e^{\gamma+\sigma}, \quad \operatorname{Re} U=e^{\gamma-\sigma} .
$$

We have $a=e^{\gamma_{(0)}}, A=e^{\sigma_{(0)}}$. At the tree level, the functions $\gamma_{(0)}$ and $\sigma_{(0)}$ are constants, and $\partial \gamma, \partial \sigma$ are $O(\epsilon)$.

Let us consider the gauge part of the action in the holomorphic section associated with the heterotic string compactification. The gauge terms can be also written as

$$
\operatorname{Im} \hat{N}_{I J} \hat{\mathcal{F}}_{\mu \nu}^{I} \hat{\mathcal{F}}^{\mu \nu J}+\frac{1}{\sqrt{-g}} \operatorname{Re} \hat{N}_{I J} \hat{\mathcal{F}}_{\mu \nu}^{* I} \hat{\mathcal{F}}^{\mu \nu J}
$$

The gauge couplings we need are calculated using (13) and the full prepotential (10) and are presented in Appendix B.

Since at the one-loop level only magnetic fields are present, the second term in (24) is zero. At the one-loop level, this term can appear only if the electric fields with the charges of order $O(\epsilon)$ are generated. Since only the couplings $N_{0 i}$ contain real parts and these are of order $O(\epsilon)$, 
the (topological) terms proportional to $\operatorname{Re} N_{0 i}$ are of order $O\left(\epsilon^{2}\right)$. To conclude, all the terms in the gauge part of the action, which are absent at the tree level, at the one-loop level are of order $O\left(\epsilon^{2}\right)^{3}$.

At the tree level, only the couplings $\hat{N}_{I I}$ are non-vanishing. In the case of magnetic black hole, the relevant tree-level couplings in the "heterotic" basis associated with the heterotic string compactification are

$$
\hat{N}_{00}^{(0)}=S T U=e^{-\phi} G_{11}, \quad \hat{N}_{11}^{(0)}=S(T U)^{-1}=e^{-\phi} G^{11},
$$

and are equal to those in the action (8). At the one-loop level, there appear non-diagonal couplings and $O(\epsilon)$ corrections to the diagonal couplings (see Appendix B).

The loop-corrected gauge term $\operatorname{Im} \hat{N}_{I J} \hat{\mathcal{F}}_{\mu \nu}^{I} \hat{\mathcal{F}}^{\mu \nu J}$ written explicitly with the gauge couplings $\hat{N}_{I J}$ presented in Appendix B, is

$$
L_{g}=-2\left[\left(e^{-\phi+2 \gamma}-\epsilon \frac{n+2 v}{4}\right)\left(\hat{\mathcal{F}}^{0}\right)^{2}+\left(e^{-\phi-2 \gamma}-\epsilon \frac{n+2 v}{4} e^{-4 \gamma}\right)\left(\hat{\mathcal{F}}^{1}\right)^{2}+\epsilon \frac{n+2 v}{2} e^{-2 \gamma}\left(\hat{\mathcal{F}}^{0} \hat{\mathcal{F}}^{1}\right)\right](25
$$

The functions $v=T U V(T, U)$ and $n$ are defined in Appendix B. Identification of the fields in (19) and (25) is as in (14):

$$
F^{(1) 1}=\sqrt{8} \hat{\mathcal{F}}^{0}, \quad F_{1}^{(2)}=\sqrt{8} \hat{\mathcal{F}}^{1}
$$

the factor $\sqrt{8}$ is due to different normalizations of the actions.

General ansatz for the spherically-symmetric metric is

$$
d s_{4}^{2}=-e^{\nu} d t^{2}+e^{\lambda} d r^{2}+e^{\mu} d \Omega_{2}^{2}
$$

In the leading order, from (21) the metric components, dilaton and moduli are

$$
\begin{aligned}
\nu_{(0)} & =-\ln f_{0}, \quad \lambda_{(0)}=\ln f_{0}, \quad \mu_{(0)}=\ln f_{0}+2 \ln r, \quad \phi_{(0)}=\ln f_{0}, \\
e^{\gamma_{(0)}} & \equiv e^{\gamma_{0}}=a A, \quad e^{\sigma_{(0)}} \equiv e^{\sigma_{0}}=\frac{a}{A} .
\end{aligned}
$$

The tree-level field strengths are those in (23), and

$$
F_{(0)}^{2}=2 q^{\prime 2}, \quad q^{\prime} \equiv \frac{f_{0}^{\prime}}{f_{0}} .
$$

In the first order in $\epsilon$, we look for a solution in the form

$$
\begin{aligned}
& \nu=-\ln f_{0}+\epsilon n, \quad \lambda=\ln f_{0}+\epsilon l, \quad \mu=\ln f_{0}+2 \ln r+\epsilon m, \quad \phi=\ln f_{0}+\epsilon \varphi, \\
& \gamma=\gamma_{0}+\epsilon \gamma_{1}, \quad \sigma=\sigma_{0}+\epsilon \sigma_{1} .
\end{aligned}
$$

Here $n, m, l, \phi, \gamma_{1}$ and $\sigma_{1}$ are unknown functions which are determined from the field equations.

The system of Maxwell equations and Bianchi identities for the gauge field strengths obtained from the action $(24)$ is

$$
\partial_{\mu}\left(\sqrt{-g} I m \hat{N}_{I J} \hat{\mathcal{F}}^{J}+\operatorname{Re} \hat{N}_{I J} \hat{\mathcal{F}}^{* J}\right)^{\mu \nu}=0
$$

\footnotetext{
${ }^{3}$ This refers also to electric fields which can appear with the charges of order $O(\epsilon)$, see below.
} 
and

$$
\partial_{\mu} \hat{\mathcal{F}}^{* J \mu \nu}=0
$$

Let us consider Eq.(30) with $I=0$. The $\nu=0$ component of this equation is

$$
\partial_{r}\left(\sqrt{-g} I m \hat{N}_{0 I} \mathcal{F}^{I}+\operatorname{Re} \hat{N}_{0 I} \hat{\mathcal{F}}^{I}\right)^{0 r}=0
$$

At the tree level, $\hat{\mathcal{F}}^{0,10 r}=0$, and (32) is satisfied identically. At the one-loop level, noting that $\operatorname{Im} \hat{N}_{00}=O(1)$ and $\operatorname{Im} \hat{N}_{0 i}=O(\epsilon)$, keeping only the terms of the highest order in $\epsilon$, we obtain solution of (32) in the form

$$
\hat{\mathcal{F}}^{00 r}=\frac{\epsilon c_{0}(\vartheta, \varphi)}{\sqrt{-g^{\prime}} \operatorname{Im} \hat{N}_{00}},
$$

where $-g^{\prime}(r)=e^{\nu+\lambda+2 \mu}$ and $c_{0}(\vartheta, \varphi)$ is an arbitrary function. Bianchi identity (31) shows that $c_{0}=$ Const. In the same way, the equation with $I=1$ yields

$$
\hat{\mathcal{F}}^{01 r}=\frac{\epsilon c_{1}}{\sqrt{-g^{\prime}} \operatorname{Im} \hat{N}_{11}} .
$$

The $\nu=\vartheta$ component of Eq.(30) with $I=0$ (the $\varphi$ component yields the same result) with the accuracy of the terms of order $O(\epsilon)$ is

$$
\partial_{\vartheta}\left(\sqrt{-g} \operatorname{Im} \hat{N}_{00} \hat{\mathcal{F}}^{0}+\operatorname{Im} \hat{N}_{01} \hat{\mathcal{F}}^{1}\right)^{\vartheta \varphi}=0,
$$

and for spherically-symmetric fields is satisfied identically. Here we kept only the terms of the leading and the first orders in $\epsilon$. Similar equation holds for $I=1$. Bianchi identities are

$$
\partial_{r} \hat{\mathcal{F}}_{\vartheta \varphi}^{0,1}=0
$$

implying that the field strengths have the form

$$
\hat{\mathcal{F}}_{\varphi \vartheta}^{0,1}=P^{0,1} \sin \vartheta
$$

Comparing with the field strengths (23) and (26), we find that

$$
P^{0}=\frac{e^{-\gamma_{0}} P}{\sqrt{8}}, \quad P^{1}=\frac{e^{\gamma_{0}} P}{\sqrt{8}} .
$$

The fields $\hat{\mathcal{F}}^{00 r}$ and $\hat{\mathcal{F}}^{10 r}$, being of order $O(\epsilon)$, contribute to the Einstein and dilaton equations the terms of order $O\left(\epsilon^{2}\right)$.

Keeping in the dilaton equation of motion the terms up to the order $O(\epsilon)$, we obtain

$$
\frac{1}{\sqrt{-g}} \partial_{\mu}\left(g^{\mu \nu} \sqrt{-g} \partial_{\nu} \phi\right)+\frac{1}{4}\left(e^{-\phi+2 \gamma}\left(F^{(1) 1}\right)^{2}+e^{-\phi-2 \gamma}\left(F_{1}^{(2)}\right)^{2}\right)=0
$$

The terms with $\partial \sigma$ and $\partial \gamma$ are of the next order on string coupling and are omitted. With the required accuracy, the terms with the gauge fields can be written as

$$
\frac{1}{4} e^{-\phi}\left[e^{2 \gamma_{0}}\left(1+2 \epsilon \gamma_{1}\right)\left(F^{(1) 1}\right)^{2}+e^{2 \gamma_{0}}\left(1-2 \epsilon \gamma_{1}\right)\left(F_{1}^{(2)}\right)^{2}\right]
$$


In view of (33), the terms with $\gamma_{1}$ cancel, and we are left with

$$
\frac{1}{\sqrt{-g}} \partial_{\mu}\left(g^{\mu \nu} \sqrt{-g} \partial_{\nu} \phi\right)+\frac{1}{2} e^{-\phi} F^{2}=0 .
$$

With the required accuracy the Einstein equations can be written as

$$
R_{\mu \nu}-\frac{1}{2} g_{\mu \nu} R-\frac{1}{2}\left(\partial_{\mu} \phi \partial_{\nu} \phi-\frac{1}{2} g_{\mu \nu}(\partial \phi)^{2}\right)+\left(L_{g}\right)_{\mu \nu}-\frac{1}{2} g_{\mu \nu} L_{g}=0
$$

Here

$$
L_{g}=\frac{1}{2} e^{-\phi} F^{2}, \quad\left(L_{g}\right)_{\mu \nu}=\frac{1}{2} e^{-\phi}\left(F^{2}\right)_{\mu \nu} .
$$

The field strengths squared have the following nonzero components

$$
\left(F^{2}\right)_{\varphi}^{\varphi}=\left(F^{2}\right)_{\vartheta}^{\vartheta}=\frac{F^{2}}{2}
$$

where

$$
\left.F^{2}=2 q^{2}(1-2 \epsilon m)\right) .
$$

The functions $\sigma_{1}$ and $\gamma_{1}$ decouple from the above equations. Since in this paper we are interested in the form of loop-corrected 4D metric and dilaton, we shall not determine these functions explicitly ( the corresponding equations of motion are solved elsewhere [10] ).

The Einstein equations (36) (with one index lifted) take the form [26]:

$$
\begin{gathered}
e^{-\lambda}\left(\mu^{\prime \prime}+\frac{3}{4} \mu^{\prime 2}-\frac{\mu^{\prime} \lambda^{\prime}}{2}\right)-e^{-\mu}+\frac{1}{4} e^{-\lambda} \phi^{2}+\frac{1}{4} e^{-\phi} F^{2}=0, \\
e^{-\lambda}\left(\frac{\mu^{\prime 2}}{2}+\mu^{\prime} \nu^{\prime}\right)-2 e^{-\mu}-\frac{1}{2} e^{-\lambda} \phi^{\prime 2}+\frac{1}{2} e^{-\phi} F^{2}=0, \\
e^{-\lambda}\left(2 \mu^{\prime \prime}+2 \nu^{\prime \prime}+\mu^{\prime 2}+\nu^{\prime 2}-\mu^{\prime} \lambda^{\prime}-\nu^{\prime} \lambda^{\prime}+\mu^{\prime} \nu^{\prime}\right)+e^{-\lambda} \phi^{\prime 2}-e^{-\phi} F^{2}=0 .
\end{gathered}
$$

With the required accuracy, in the equations of motion, all the expressions of the first order in string coupling $\epsilon$ are calculated with the tree-level moduli.

\section{Loop-corrected metric and dilaton}

To solve the loop-corrected equations of motion with the required accuracy, i.e. in the first order in the loop-counting parameter $\epsilon$, we substitute in the above equations the functions $\mu, \nu, \lambda$ and $\phi$ in the form (29) In Appendix A we present the detailed solution of the equations of motion in the ansatz $l=-n^{4}$.

$$
n=\frac{P}{r}\left(A_{1}-\frac{C_{2}}{2}\right)-A_{2} \frac{P}{r+P} \quad m=-\frac{P}{r} A_{1}+A_{2} \frac{P}{r+P}
$$

\footnotetext{
${ }^{4}$ There are four equations (35) and (38)-(40) for three unknown functions $m, n$ and $\varphi$. Our choice corresponds to the requirement that in the first order in $\epsilon$, as in the leading order, $\nu=-\lambda$.
} 
and

$$
\varphi=\frac{P}{r} A_{1}+A_{2} \frac{P}{r+P} .
$$

Here $A_{1}, A_{2}$ and $C_{2}$ are arbitrary constants.

By coordinate transformation the metric (27) with $\nu=-\lambda$ can be reduced to the form

$$
d s^{2}=-e^{U(R)} d t^{2}+e^{-U(R)}\left(d R^{2}+R^{2} d \Omega^{2}\right)
$$

where the new variable $R$ is determined from the relation

$$
\ln \frac{R}{R_{1}}=\int_{r_{1}}^{r} d r^{\prime} e^{\frac{1}{2}(\lambda-\mu)\left(r^{\prime}\right)}
$$

For the solution (41), we have $R=C e^{\frac{\epsilon C_{2}}{2 r}}$, where $C$ is an arbitrary constant. In new coordinates, the asymptotics of the metric and dilaton are obtained by setting in (41) $C_{2}=0$.

The ADM mass calculated with the metric (43) is

$$
M=2 P\left(1+\epsilon A_{1}\right) .
$$

The expressions (41)-(42) were obtained by making the expansions of $e^{\epsilon n}, e^{\epsilon l}$, etc. to the first order in $\epsilon$ assuming that $1>|\epsilon n|,|\epsilon l|, \ldots$. This yields

$$
r>\epsilon P\left(\left|A_{1}\right|+\left|A_{2}\right|\right) .
$$

Solving the system of the Einstein-Maxwell equations and the equations of motion for the moduli, we cannot decide which solution is supersymmetric. This issue can be solved by studying the $N=2$ supersymmetry transformations and solving Killing spinor equations [10]. We found that the supersymmetric solution with the metric of the form (43) corresponds to

$$
A_{1}=P|h| T U / 2
$$

(in notations of Sect.4 $T U=G_{11}=e^{2 \gamma_{0}}=a^{2}$ ). From the explicit form of the prepotential which was calculated in [19] for the case of unbroken gauge group is $\left[E_{8} \times E_{7} \times U(1)^{2}\right]_{L} \times U(1)_{R}^{2}$ follows that $h$ is negative ${ }^{5}$. and the loop-corrected metric is

$$
g^{i i}=-g_{00}=\frac{r}{r+P}\left(1+\epsilon \frac{P|h| / 2 T U}{r}\right) \text {. }
$$

Extrapolating this expression to the region of of small $r$, we note that the singularity at $r=0$ is smeared by quantum corrections.

\footnotetext{
${ }^{5}$ Strictly speaking, in the above expressions for the gauge couplings above stands $R e h$. Prepotential $h$ is defined up to a quadratic polynomial in $T, U$ and $T U$ with imaginary coefficients. Although the gauge couplings contain the ambiguities, these cancel in the expressions for the field strengths, and the final results are unambiguous [10]
} 


\section{Discussion}

In this paper we discussed solutions to the equations of motion of the string-loop-corrected $4 D, \quad N=2$ effective action obtained by dimensional reduction on a two-torus from the $6 D, \quad N=1$ effective action of heterotic string theory. The effective action contains both the dilaton and the metric of compact dimensions. As a tree-level solution, we considered the magnetic black hole solution.

Different embeddings of $N=2 S T U$ model in type IIA and IIB theories and construction of the generating solution of regular BPS tree-level black hole solutions were studied in [31] and refs. therein. Embeddings with charges stemming from R-R and NS-NS sectors are possible. In the case of heterotic embedding considered in the present paper the generating solution is the same as in the case of non-symmetric NS-NS embedding of the STU model in toroidally compactified type II theory. From the point of view of the perturbation theory, the above embedding is singled out, because in this case dilaton is one of the moduli, and the dilatonaxion parametrize a separate factor of the moduli space.

Our discussion was simplified by considering the tree-level solution with equal magnetic charges, in which case the string-loop corrections are independent of coordinates, although our treatment can be extended to the case of unequal charges.

Let us discuss modification of the above results in the case of unequal charges $P_{1} \neq P_{2}$ (for simplicity we set in (22) $a=A=1$ ). We are mainly interested in the small- $r$ region. The tree-level expressions are modified in the following way: the functions $\mu_{(0)}, \nu_{(0)}, \lambda_{(0)}$ and $\phi_{(0)}$ retain the same functional form (28) as at the tree level, except for the function $f_{0}$ which is changed to

$$
\tilde{f}_{0}=\left[\left(1+\frac{P_{1}}{r}\right)\left(1+\frac{P_{2}}{r}\right)\right]^{1 / 2} .
$$

The squares of field strengths are

$$
F^{(i)^{2}}=2 P_{i}^{2} \frac{1}{\tilde{f}_{0}^{2} r^{4}} .
$$

For the metric components of the internal two-torus we obtain

$$
G_{11}=\frac{P_{1}+r}{P_{2}+r}=\frac{P_{1}}{P_{2}}+r\left(\frac{1}{P_{2}}-\frac{1}{P_{1}}\right)+O\left(r^{2}\right), \quad G_{22}=1 .
$$

The modulus $\gamma$ is now $r$-dependent, and at small $r$ we have

$$
\partial_{r} \gamma=a+\epsilon \partial_{r} \gamma_{1}, \quad a=\frac{1}{2}\left(\frac{1}{P_{2}}-\frac{1}{P_{1}}\right) .
$$

Except for a substitution of $f_{0}$ by $\tilde{f}_{0}$, in the gauge action there appear new terms of order $O(\epsilon)$

$$
(\partial \gamma)^{2} \epsilon e^{\phi} F_{\gamma \gamma}+2 \partial \phi \partial \gamma \epsilon e^{\phi} F_{\phi \gamma},
$$

where the functions $F_{\gamma \gamma}$ and $F_{\phi \gamma}$ are expressed via the derivatives of the prepotential.

In the limit $r \rightarrow 0$, the $O(\epsilon)$ terms with the most singular behavior stemming from the gauge and dilaton parts of the action are

$$
\epsilon \frac{v}{2} e^{-2 \gamma} P_{1} P_{2} e^{-\phi} \frac{1}{\tilde{f}_{0}^{2} r^{4}}
$$


and

$$
\epsilon(\partial \phi)^{2} v e^{-2 \gamma} e^{\phi}=-\epsilon{\tilde{q^{\prime}}}^{2} v e^{-2 \gamma} e^{\phi},
$$

where $\tilde{q}^{\prime}=\frac{\tilde{f}_{0}^{\prime}}{\tilde{f}_{0}}$. At small $r$, these terms retain the same functional form as in the case $P_{1}=P_{2}$, because ${\tilde{q^{\prime}}}^{2}=\frac{P_{1} P_{2}}{\tilde{f}_{0}^{2} r^{4}}\left(1+O\left(r^{2}\right)\right)$.

As a result, in the case of unequal charges the equations of motion retain the same functional form as for equal charges, except for the extra terms of order $\epsilon a \tilde{q^{\prime}} e^{\phi}$, where $a$ is defined in (47). Solving the equations of motion along the lines of Appendix 1, we find that solutions obtained in the case of equal charges are modified by terms of order $O(a \ln r)$ which at small $r$ are much smaller than the leading terms of order $\frac{1}{r}$.

Another way to obtain the loop-corrected expressions for the metric and moduli is to solve the spinor Killing equations for the $N=2$ supersymmetric loop-corrected effective action. Details of this work are reported elsewhere [10].

Perturbative expansion in string coupling implies that the non-perturbative corrections of the form $O\left(e^{-2 \pi S}\right)$ are absent. This means that in the weak coupling limit we consider, we cannot check duality properties of the full theory which are restored only in the full nonperturbative setting [28].

The problem of string-loop corrections to the classical charged black holes in the effective $N=2$ supergravity was studied also in papers $[29,30]$. However, the two approaches are rather different. In the these papers, the loop corrections were calculated under an assumption that there exists a "small" modulus and it is possible to expand the loop correction to the prepotential with respect to the ratios of small to large moduli. Within the framework of string perturbation theory, the natural expansion parameter is connected with the dilaton, but this modulus does not enter the loop correction to the prepotential. The remaining two moduli connected with the metric of the compact two-torus for special configurations may have parametric smallness, but not the functional one connected with dependence on $r$. Moreover, as we have argued above, to study the loop corrected solution it is important to take into account corrections to the gauge couplings.

The string-tree-level chiral null model provides a solution to the low energy effective action which, in a special renormalization scheme, receives no $\alpha^{\prime}$ corrections [5, 12]. The loopcorrected solution is no longer expressed in terms of harmonic functions, and the $\alpha^{\prime}$-corrections are present. However, still it is possible that $\alpha^{\prime}$ corrections are small and can be treated perturbatively. It may be noted here that smearing of the singularity of the point-like source by $\alpha^{\prime}$-corrections was discussed previously in [32].

\section{Acknowledgments}

I would like to thank R. Kallosh for helpful correspondence, A. Marshakov, O. Kechkin, A. Sagnotti and I.Tyutin for remarks and discussion.

The work of M.I. was partially supported by the RFFR grant No 00-02-17679. 


\section{A Solution of field equations}

In the first order in parameter $\epsilon$ the Einstein equations (38)-(40) are ${ }^{6}$

$$
\begin{gathered}
m^{\prime \prime}+m^{\prime}\left(q^{\prime}+\frac{3}{r}\right)-l^{\prime}\left(\frac{1}{2} q^{\prime}+\frac{1}{r}\right)+\varphi^{\prime} \frac{q^{\prime}}{2}-\frac{l-m}{r^{2}}+\frac{1}{2} q^{2} s=0, \\
m^{\prime} \frac{2}{r}+n^{\prime}\left(q^{\prime}+\frac{2}{r}\right)-\varphi^{\prime} q^{\prime}-2 \frac{l-m}{r^{2}}+q^{\prime 2} s=0, \\
m^{\prime \prime}+n^{\prime \prime}+m^{\prime} \frac{2}{r}-l^{\prime} \frac{1}{r}+n^{\prime}\left(-q^{\prime}+\frac{1}{r}\right)+\varphi^{\prime} q^{\prime}-q^{\prime 2} s=0 .
\end{gathered}
$$

Here

$$
s=l-\varphi-2 m
$$

We also need the equation for the dilaton $(35)$ in the $O(\epsilon)$ order:

$$
\varphi^{\prime \prime}+\frac{2}{r} \varphi^{\prime}+\frac{1}{2}\left(2 m^{\prime}+n^{\prime}-l^{\prime}\right) q^{\prime}+q^{\prime 2} s+a f_{0} q^{2}=0 .
$$

We look for a solution such that $l=-n$, because in this case, as at the tree level, the components of the metric satisfy the relation $g_{t t}=g_{r r}^{-1}$. Substituting this ansatz in the above equations and forming the combination of equations $(48)-\frac{1}{2}(49)$, we have

$$
m^{\prime \prime}+m^{\prime}\left(q^{\prime}+\frac{2}{r}\right)+\varphi^{\prime} q^{\prime}=0 .
$$

Taking into account the explicit form of the functions $f_{0}$ and $q^{\prime}=\frac{f_{0}{ }^{\prime}}{f_{0}}$, we integrate this equation and obtain the first integral as

$$
m^{\prime}+q^{\prime}\left(C_{1}+\varphi\right)=0,
$$

where $C_{1}$ is an integration constant. To solve Eqs.(49) and (50) we introduce the combinations $u=m+n$ and $t=n-\varphi$. The function $s$ in (51) is

$$
s=-2 u+t .
$$

Esq.(49) and (50) take the form

$$
\begin{aligned}
u^{\prime} \frac{2}{r}+t^{\prime} q^{\prime}+2 \frac{u}{r^{2}}+q^{\prime 2} s & =0 \\
u^{\prime \prime}+u^{\prime} \frac{2}{r}-t^{\prime} q^{\prime}-q^{\prime 2} s & =0 .
\end{aligned}
$$

The sum of these equations is

$$
u^{\prime \prime}+u^{\prime} \frac{4}{r}+u \frac{2}{r^{2}}=0
$$

yielding solution $u=\frac{C_{2}}{r}+\frac{C_{2}{ }^{\prime}}{r^{2}}$. We set $C_{2}{ }^{\prime}=0$ to avoid a solution with a too rapid growth of the function at small $r$. Substituting $u=\frac{C_{2}}{r}$ in Eq. (55) (either equation yields the same result), we arrive at the equation

$$
t^{\prime}+q^{\prime} t=q^{\prime} \frac{2 C_{2}}{r}
$$

\footnotetext{
${ }^{6}$ Below we work with dimensionless variable $r$. In the final expressions $P$ is reinstated by substitution $r \rightarrow \frac{r}{P}$.
} 
with the solution

$$
t=C_{3}+\frac{C_{2}}{r}-\frac{C_{2}+C_{3}}{r+1} .
$$

Substituting $\varphi=-m+u-t$ in (54), we solve this equation and obtain

$$
m=-\frac{1}{r}\left(C_{1}+\frac{C_{2}}{2}-\frac{C_{3}}{2}-C_{4}\right)+\frac{1}{r+1}\left(\frac{C_{2}}{2}+\frac{C_{3}}{2}\right)+C_{4} .
$$

Using (58) for $t$, (59) for $m$, and $u=\frac{C_{2}}{r}$, we have

$$
\varphi=\frac{1}{r}\left(C_{1}+\frac{C_{2}}{2}-\frac{C_{3}}{2}-C_{4}\right)+\frac{1}{(r+1)}\left(\frac{C_{2}}{2}+\frac{C_{3}}{2}\right)-C_{3}-C_{4} .
$$

Eq. (A5) with $m+n=u=\frac{C_{2}}{r}$ and $s=-2 u+t$ with $t$ from (A11) yields

$$
\varphi^{\prime}=\left(\frac{C_{2}}{2}+\frac{C_{3}}{2}\right)\left(\frac{1}{r^{2}}-\frac{1}{(r+1)^{2}}\right)+\frac{C_{5}}{r^{2}} .
$$

Comparing (61) with the derivative of the solution (60), we find that the coefficients at the terms $(r+1)^{-2}$ coincide, and coefficients at the terms $r^{-2}$ yield the relation expressing the constant $C_{5}$ in terms of other constants.

Next we require that in the limit $r \rightarrow \infty$ our solutions should be asymptotic to the Lorentzian metric. This requirement sets $C_{3}=C_{4}=0$ and we are left with

$$
\begin{aligned}
\varphi & =\frac{1}{r}\left(C_{1}+\frac{C_{2}}{2}\right)+\frac{1}{r+1} \frac{C_{2}}{2}, \\
m & =-\frac{1}{r}\left(C_{1}+\frac{C_{2}}{2}\right)+\frac{1}{r+1} \frac{C_{2}}{2}, \\
n & =\frac{1}{r}\left(C_{1}-\frac{C_{2}}{2}\right)-\frac{1}{r+1} \frac{C_{2}}{2} .
\end{aligned}
$$

Introducing new constants

$$
A_{1}=C_{1}-\frac{C_{2}}{2}
$$

and

$$
A_{2}=\frac{C_{2}}{2}
$$

we obtain the expressions discussed in Sect.5.

\section{B Loop-corrected gauge couplings}

We remind the notations: $\frac{X^{i}}{X^{0}}=i y_{i}$, where

$$
\left(y_{1}, y_{2}, y_{3}\right)=(S, T, U)
$$

and consider real $S, T, U$ relevant to this paper. The prepotential $\mathcal{F}=-i X^{0^{2}} F(X)$ is

$$
\mathcal{F}(z)=y_{1} y_{2} y_{3}+\epsilon h\left(y_{2}, y_{3}\right)
$$


where $h\left(y_{1}, y_{2}\right)$ is a real function. Define $h_{i} \partial y_{j}$ and

$$
\begin{gathered}
v=h-y_{i} h_{i}, \\
n=h-y_{i} h_{i}+y_{i} h_{i j} y_{j} .
\end{gathered}
$$

The function $v$ is connected with the Green-Schwarz function $V$ introduced in Sect.3 as

$$
v(T, U)=V(T, U) T U=h^{(1)}-T \partial_{T} h^{(1)}-U \partial_{U} h^{(1)}
$$

Because the functions $V$ and $v$ appear in the first order in the string coupling $\epsilon$, they are calculated with the tree-level moduli. In particular, we have $V=v e^{-2 \gamma_{0}}$.

In the basis admitting a prepotential, the gauge couplings are calculated using the formula (13), and we have (we list only those used in our calculations)

$$
\begin{aligned}
& N_{00}=-i\left(y_{1} y_{2} y_{3}-\epsilon \frac{n}{4}\right), \\
& N_{01}=-\epsilon \frac{n+2 v}{4 y_{1}}+i \epsilon a_{1} \frac{y_{2} y_{3}}{y_{1}} \\
& N_{11}=-i \frac{y_{2} y_{3}}{y_{1}}\left(1+\epsilon \frac{n}{4 y_{1} y_{2} y_{3}}\right) .
\end{aligned}
$$

The couplings in the "heterotic" basis are obtained via the transformation (20). In the first order in $\epsilon$ we obtain

$$
\begin{aligned}
& \hat{N}_{00}=N_{00}=-i y_{1} y_{2} y_{3}\left(1-\epsilon \frac{n}{4 y_{1} y_{2} y_{3}}\right)=-i\left(e^{-\phi+2 \gamma}-\epsilon \frac{n}{4}\right), \\
& \hat{N}_{01}=-\frac{N_{01}}{N_{11}}=-i \epsilon \frac{n+2 v}{y_{2} y_{3}}+\epsilon a_{1}=i \epsilon e^{-2 \gamma}(n+2 v)+\epsilon a_{1}, \\
& \hat{N}_{11}=-\frac{1}{N_{11}}=-i \frac{y_{1}}{y_{2} y_{3}}\left(1-\epsilon \frac{n}{4 y_{1} y_{2} y_{3}}\right)=-i\left(e^{-\phi-2 \gamma}-\epsilon \frac{n}{4} e^{-4 \gamma}\right) .
\end{aligned}
$$

The Green-Schwarz function $V(T, \bar{T}, U, \bar{U})$ was estimated numerically in [11] and was shown to be positive. Note also that for small $T-U$, i.e. near the points of enhanced symmetry $T=U, h^{(1)}(T, U)=-\frac{1}{16 \pi^{2}}(T-U)^{2} \log (T-U)^{2}+$ regular, where the regular term is finite for $T \approx U \neq 1, e^{\pi i / 6}$. The second derivatives of the function $h^{(1)}(T, U)$ at the points of the enhanced symmetry have logarithmic singularities. To avoid these points, we introduced in (22) the constants $a$ and $A$ choosing them so that they are not connected by a rational function thus ensuring that the moduli cannot be transformed to each other by a modular transformation. However, all final expressions depend only on the function $V$, which contains only the first derivatives of the prepotential and is non-singular for all values of $T$ and $U$. Thus, our expressions are valid for any $a$ and $A$.

\section{References}

[1] M. Green, J. Schwarz and E. Witten, Superstring Theory, Cambridge University Press, 1987. 
[2] D. Youm, Phys. Rept. 316, 1 (1999), hep-th/9710046.

[3] A. W. Peet, Class. Quant. Grav. 15, 3291 (1998), hep-th/9712253.

[4] G. T. Horowitz and A. A. Tseytlin, Phys. Rev. D51, 2896 (1995), hep-th/9409021; A. A. Tseytlin, Mod. Phys. Lett. A11, 689 (1996), hep-th/9601177.

[5] M. Cvetic and A. A. Tseytlin, Phys. Lett. B366, 95 (1996), hep-th/9510097; Phys. Rev. D53, 5619 (1996), hep-th/9512031.

[6] I. Antoniadis, S. Ferrara, E. Gava, K. S. Narain and T. R. Taylor, Nucl. Phys. B447, 35 (1995), hep-th/9504034.

[7] B. de Wit, V. Kaplunovsky, J. Louis and D. Luest, Nucl. Phys. B451, 53 (1995), hepth/9504006.

[8] J. P. Derendinger, S. Ferrara, C. Kounnas and F. Zwirner, Nucl. Phys. B372, 145 (1992).

[9] G. L. Cardoso, B. de Wit, J. Kappeli and T. Mohaupt, JHEP, 0012, 019 (2000), hepth/0009234; T. Mohaupt, Fortsch. Phys., 49, 3 (2001), hep-th/0007195.

[10] M.Z. Iofa, hep-th/0104163.

[11] E. Kiritsis, C. Kounnas, P. M. Petropoulos and J. Rizos, Nucl. Phys B483, 141 (1997), hep-th/9608034.

[12] P. Howe and G. Papadopoulos, Nucl. Phys. B289, 264 (1987); ibid. B381, 360 (1992).

[13] A. Sen, Int. J. Mod. Phys. A9, 3707 (1994), hep-th/9402002.

[14] M. Cvetic and D. Youm, Nucl. Phys. B453, 259 (1995), hep-th/9505045; Phys. Rev. D53, 584 (1996), hep-th/9507090.

[15] B. de Wit and A. Van Proyen, Nucl. Phys. B245, 89 (1984); B. de Wit, P. Lauwers and A. Van Proyen, Nucl. Phys. B255, 569 (1985).

[16] A. Ceresole, R. D'Auria, S. Ferrara and A. Van Proeyen, Nucl. Phys. B444, 92 (1995, hep-th/9502072.

[17] L. Andrianopoli et. al., J. Geom. Phys. 23, 111 (1997), hep-th/9605032.

[18] B. Craps, F. Roose, W. Troost and A. Van Proeyen, Nucl. Phys. B503, 565 (1997), hep-th/9703082.

[19] J. A. Harvey and G. Moore, Nucl. Phys. B463, 315 (1996), hep-th/9510182.

[20] R. D'Auria, S. Ferrara and P. Fre, Nucl. Phys. B359, 705 (1991).

[21] M. J. Duff, J. T. Liu and J. Rahmfeld, Nucl. Phys. B459, 125 (1996), hep-th/9508094.

[22] K. Behrndt et. al., Phys. Rev. D54, 6293 (1996), hep-th/9608059. 
[23] V.S. Kaplunovsky, Nucl. Phys. B307, 145 (1988), hep-th/9205070; L. Dixon, V.S. Kaplunovsky and J. Louis, Nucl. Phys. B355, 649 (1991).

[24] I. Antoniadis, K. S. Narain and T.R. Taylor, Phys. Lett., B267, 37 (1991); I. Antoniadis, E. Gava and K. S. Narain, Nucl. Phys., B283, 93 (1992).

[25] V.S. Kaplunovsky and J. Louis, Nucl. Phys B444, 191 (1995), hep-th/9502077.

[26] L.D. Landau and E.M. Lifshitz, Field Theory, Nauka, Moscow, 1985.

[27] J. X. Lu, Phys. Lett. B313, 29 (1993), hep-th/9304159.

[28] G. L. Cardoso et. al., Nucl. Phys. B464, 18 (1996), hep-th/9512129; G. L. Cardoso et. al. Phys. Lett. B382, 241 (1996), hep-th/9603108.

[29] K. Behrndt, D. Lust and W. A. Sabra, Nucl.Phys. B510, 264 (1998), hep-th/9705169.

[30] K. Behrndt and I. Gaida, Phys. Lett. B401, 263 (1997), hep-th/9702168; K. Behrndt, G. Lopes Cardoso and I. Gaida, Nucl. Phys.B506, 267 (1997), hep-th/9704095.

[31] M. Bertolini, P. Fre and M. Trigiante, Class. Quant. Grav. 16, 1519 (1999), hepth/9811251; M. Bertolini and M. Trigiante, Int. J. Mod. Phys. A15, 5017 (2000), hepth/9910237; Nucl. Phys. B582, 393 (2000), hep-th/0002191.

[32] A.A. Tseytlin, Phys. Lett. B363, 223 (1995), hep-th/9509050. 